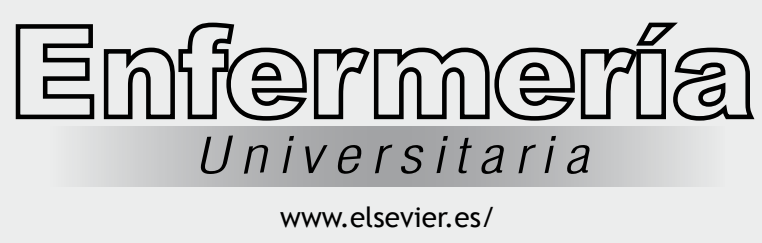

ARTÍCULO DE INVESTIGACIÓN

\title{
Experiencias y conocimientos de los estudiantes de enfermería, ante la muerte del paciente hospitalizado
}

\author{
M. Á. Orozco-Gonzáleza, G. 0. Tello-Sáncheza , R. Sierra-Aguillón ${ }^{b}$, R. M. Gallegos- \\ Torres $^{\text {b, }}$, Á. S. Xeque-Morales ${ }^{b}$, B. L. Reyes-Rocha ${ }^{\mathrm{b}}$ y A. Zamora-Mendoza ${ }^{\mathrm{b}}$
}

a Pasantes de la Licenciatura en Enfermería, Facultad de Enfermería, Universidad Autónoma de Querétaro, Querétaro, Qro., México

b Facultad de Enfermería, Universidad Autónoma de Querétaro, Querétaro, Qro., México

Recibido: 10 octubre 2012; Aceptado: 13 diciembre 2012

\section{PALABRAS CLAVE \\ Enfermería; \\ Educación; Muerte; \\ Experiencias; \\ Estudiantes; México.}

\begin{abstract}
Resumen
Objetivo: Identificar conocimientos y experiencias de estudiantes de Licenciatura en Enfermería, sobre la muerte de pacientes hospitalizados.

Métodos: Estudio transversal, descriptivo, exploratorio, en el que participaron 188 alumnos de 500 , pertenecientes a $4^{\circ}, 6^{\circ}$ y $8^{\circ}$ semestre (quienes tienen experiencia clínica). Se consideraron tanto hombres como mujeres, sin distinción de edad o experiencia previa ante la muerte. Se diseñó un instrumento ex profeso para el levantamiento de datos, el cual fue validado por expertos mediante técnica Delphi. El proyecto fue avalado por un Comité de Investigación y Bioética. Los resultados se procesaron en SPSS v. 17.

Resultados: El 85\% de los alumnos ha experimentado un proceso de muerte de un familiar o amigo, y el $75 \%$ de un paciente (en la práctica hospitalaria). El $46 \%$ no se consideran preparados para vivir esta experiencia. El 37.7\% considera que el personal de las instituciones no está preparado para "manejar" la muerte de los pacientes. Hay opiniones divididas sobre el temor hacia este fenómeno. Los alumnos comprenden qué es la tanatología y qué es el duelo, aunque no se sienten competentes en el tema. Conclusiones: Es importante sensibilizar al futuro profesional de enfermería en competencias que permitan dar un trato digno al paciente, esto fundamentado en sólidos conocimientos en tanatología.
\end{abstract}

*Autor para correspondencia: Correo electrónico: isisrmgx@gmail.com (R. M. Gallegos-Torres). 


\section{KEYWORDS}

Nursing; Education; Death experiences; Students; Mexico.

\section{Nursing students' knowledge and experiences on death of hospitalized patients}

\begin{abstract}
Objective: To identify knowledge and experiences of baccalaureate nursing students about death of hospitalized patients.

Methods: Basic, descriptive, and exploratory study on 188 students of $4^{\text {th }}, 6^{\text {th }}$, and $8^{\text {th }}$ semester with clinical practices. Both men and women, regardless of age and previous experience with death, were considered. A Delphi technique expert validated specific instrument was designed. The project was approved by the Research and Bioethics Committee. Results were processed in SPSS v. 17.

Results: The $85 \%$ of students have experienced the death of a relative or a friend, and $75 \%$ of a patient (at the hospital practice), while $46 \%$ do not consider themselves prepared to live this experience, and $37.7 \%$ consider that the institution staff are not prepared to "handle" the death of patients. There are divided opinions about the fear to this process. Although students understand tanatology and duel, they do not feel competent in the topic.

Conclusions: It is important to sensitize the future nursing professional in skills which allow them offer the corresponding appropriate patient treatment.
\end{abstract}

\section{Introducción}

En un mundo tan complejo (con problemas económicos, sociales, medioambientales, políticos y con una tecnología cada vez más abundante), no se ha favorecido que los procesos emocionales y psicológicos de los individuos sean sencillos, por el contrario, se observa a una sociedad a menudo carente de elementos familiares y personales, que los puedan llevar a enfrentar situaciones estresantes o problemáticas diversas de una manera eficaz. Tal es el caso del fenómeno de la muerte.

Estudiada desde los orígenes de la humanidad, la muerte ha sido interpretada y valorada con diferentes concepciones ${ }^{1}$. Uno de estos abordajes es el personalista, el cual, entre otras cuestiones, analiza el "hecho humano de morir, la percepción y la vivencia de la muerte humana”. Dicho enfoque, se interesa en conocer el sentido de la experiencia de la muerte desde el punto de vista de los individuos ${ }^{2}$.

Al respecto, Safatle ${ }^{3}$ indica que el concepto de muerte es relativo, ya que depende del desarrollo psíquico de cada persona y de su situación afectiva; es complejo, cambiante y depende de diversas situaciones sociales, por lo que es reconocido como un proceso.

El proceso de la muerte es un tema aun difícilmente aceptado y comprendido por gran parte de la población. Podría tal vez hacerse el señalamiento de que el personal de la salud, por estar más "en contacto" con personas que día a día fallecen, es quien está mejor preparado para vivirlo, sin embargo, es ampliamente reconocido que los involucrados en el área, no se sienten competentes al respecto, lo que ha llevado a establecer diversas premisas en torno a este fenómeno, entre las cuales se rescatan para este estudio las siguientes:

1. La muerte, como ningún otro evento de la vida, es capaz de suscitar en los seres humanos, más pensamientos y reacciones provocados por este suceso, sea en el individuo que está muriendo como en aquellos que están a su alrededor ${ }^{1}$.

2. Es necesario que los individuos posean cierta capacidad de enfrentar los "problemas de la vida" de manera coherente y lógica, de forma tal que se pueda tener una actitud digna y responsable ante dichos "problemas"2.

Así, la manera de estudiar la muerte y de abordarla, demanda una actitud de comprensión íntima y de observación externa del fenómeno ${ }^{4}$, aspecto que por su naturaleza, implica un grado de complejidad mayor, dado que difícilmente se puede tener una "experiencia" sobre ella, sin embargo, es parte de la vida humana ${ }^{2}$.

Relacionado con lo anterior, la tanatología se reconoce como la ciencia que estudia y reflexiona sobre la muerte, las relaciones del hombre con ésta y sus consecuencias ${ }^{5}$.

En el caso del personal de salud, la convivencia profesional con la experiencia de la muerte y su proceso se encuentra reconocida como una de las vivencias más impactantes para la mayor parte de sus practicantes, siendo uno de los retos más grandes que deben afrontar tanto los profesionales como los estudiantes ${ }^{6}$, ya que generalmente se les educa para considerar mucho más los aspectos biológicos que los psicológicos o espirituales de las personas ${ }^{1}$.

Tanto el médico como la enfermera conviven más frecuentemente con la muerte, pero no siempre constituye para ellos un evento que los marque positivamente ${ }^{7}$, sobre todo a la enfermera(o) dado que la muerte no sólo implica el acto físico del cese de las constantes vitales, sino que involucra todo un proceso de duelo del paciente y de las personas a su alrededor, incluso desde el momento mismo en que se conoce la posibilidad de fallecimiento.

Dentro de las instituciones hospitalarias, el profesional de enfermería, ante la muerte de un paciente, es el responsable de brindar los cuidados post mortem, entendidos éstos como la serie de atenciones que se prestan a la persona fallecida, entre las cuales están: 1) el registro de la hora del fallecimiento, 2) establecimiento de la identidad de quien falleció, 3) retiro de tubos, catéteres, drenes, etc., 4) colocación de los brazos del cuerpo cruzados sobre el tó$\operatorname{rax}^{8}$.

Sin embargo, todo profesional del área de la salud debería estar preparado anímica y profesionalmente para afrontar 
las variadas situaciones posibles en torno a la muerte de un paciente ${ }^{9,10}$.

De esta manera, si el profesional de enfermería entiende el proceso de duelo que afecta al paciente y la familia, puede apoyar a ambos y a sí mismo, a redefinir las situaciones que se generan previa y posteriormente a la muerte ${ }^{11}$, contar con un espíritu humanístico, estar sensibilizado, concientizado, familiarizado y sobre todo, capacitado para poder otorgar cuidados especializados, lo cual favorecería cumplir el compromiso ético con cada uno de los pacientes, de acompañarles, brindarles cuidados de calidad y asimismo, aliviar los síntomas adversos que se presentan durante el trayecto de su enfermedad; y en los momentos finales respetar la autonomía del paciente y permitirle que parta en compañía de sus seres queridos, en un ambiente agradable de amor y calidez ${ }^{12}$.

Sin embargo, el mecanismo psicológico más común de defensa del profesional de la salud es el "no sufrir", adoptar la actitud de "no pasa nada", es decir, adoptar la indiferencia como escudo. No perder la "profesionalidad" controlando los sentimientos generados por el fenómeno. Como regla de oro se reconoce el "no involucrarse con el paciente", "guardar distancia". Así, se identifica que 2 pueden ser las respuestas extremas ante la muerte: 1) angustia, estrés, sufrimiento, que puede llevar a un desgaste emocional y frustración; 2) actitud de indiferencia, pensar que es otro paciente más, una estadística más?

Desafortunadamente, lo anterior es llevado al terreno estudiantil "transmitido" no solamente por el docente, sino por el mismo personal clínico con el que se relacionan los alumnos, donde se generan actitudes de indiferencia ante el fenómeno, aspecto dado igualmente porque durante la formación universitaria, no se cuenta con la preparación necesaria para el manejo de las emociones y sensaciones que la primera experiencia (y posteriores), con la muerte suscita.

Al respecto, Carvalho en de Martins ${ }^{13}$, señala que es una responsabilidad para la formación en enfermería el involucramiento de la muerte con los estudiantes, evaluar la relación de éstos con la negación, ocultamiento de los sentimientos ante la muerte, entre otros, que son indicativos de una falta de preparación universitaria para la futura práctica profesional.

Relacionado con lo anterior, claramente se identifica que el conocimiento sobre un fenómeno prepara a la persona en situaciones donde requiere hacer uso de dicho conocimiento, sin embargo, en la mayoría de las ocasiones las experiencias fortalecen la información sobre un tema y por ende, el actuar, por lo que es lógico imaginar que con respecto a la muerte y a las experiencias previas de los futuros profesionistas, es necesario tener resultados de investigaciones que permitan ahondar en este aspecto.

Así, se propuso llevar a cabo un trabajo de investigación con la finalidad de identificar los conocimientos y experiencias generales que sobre la muerte de los pacientes hospitalizados, tienen los estudiantes de la Licenciatura en Enfermería de la Universidad Autónoma de Querétaro, en Querétaro, México.

\section{Métodos}

Se realizó un estudio transversal, descriptivo, exploratorio; el universo lo conformaron más de 500 estudiantes de la Licenciatura en Enfermería de la Universidad Autónoma de Querétaro, campus Ciudad Universitaria, inscritos en el año 2011; la muestra se conformó con aquellos alumnos del $4^{\circ}$, $6^{\circ}$ y $8^{\circ}$ semestre, quienes ya han tenido experiencia clínica a través de las prácticas hospitalarias. Fueron tomados en cuenta todos los alumnos que aceptaran participar en el estudio, por lo que fue un muestreo por conveniencia, sin importar edad, sexo y si tuvieron o no experiencias ante la muerte de un paciente.

Con base en la operacionalización de las variables (tabla 1), que consideró los aspectos de conocimientos y experiencias, se diseñó un instrumento ex profeso para el levantamiento de datos, el cual fue validado mediante técnica Delphi por 5 expertos en el área de la salud y psicología, con antecedentes de trabajo en investigación y nivel estudio mínimo de maestría; previo levantamiento de datos, se llevó a cabo una prueba piloto con alumnos de $9^{\circ}$ semestre; hechos los ajustes pertinentes, se conformó la versión final del instrumento, el cual se denominó “Experiencias de los estudiantes de enfermería ante la muerte”, el cual consta de 52 preguntas estructuradas, agrupadas de la siguiente manera: 1) 15 sobre experiencias generales de los estudiantes ante la muerte de pacientes hospitalizados, 2) 13 sobre conocimientos básicos de tanatología y el proceso de la muerte, 3) 16 sobre el sentir personal del morir y, 4) 8 sobre datos

Tabla 1 Operacionalización de las variables: conocimientos y experiencias

\begin{tabular}{llll}
\hline \multicolumn{1}{c}{ Variables } & \multicolumn{1}{c}{ Definición conceptual } & \multicolumn{1}{c}{ Dimensiones } & Escala de medición \\
\hline Conocimientos & $\begin{array}{l}\text { Conjunto de ideas acerca de algo o } \\
\text { cosas que se saben a ciencia cierta }{ }^{14}\end{array}$ & $\begin{array}{l}\text { Ciclo de vida } \\
\text { Aspectos básicos de tanatología } \\
\text { Ciclo del duelo }\end{array}$ & Ordinal \\
Experiencias & $\begin{array}{l}\text { Conocer o sentir alguien una cosa él } \\
\text { mismo, por sí mismo o en sí mismo. }\end{array}$ & $\begin{array}{l}\text { Sentimientos } \\
\text { Experiencias vividas }\end{array}$ & Ordinal \\
& $\begin{array}{l}\text { Suceso por los que se pasa y con los } \\
\text { que se adquiere conocimiento de la } \\
\text { vida }\end{array}$ & & \\
\end{tabular}


personales, que ayudaron a alcanzar el objetivo de identificar el nivel de conocimientos y experiencias ante la muerte, por parte de los estudiantes. El instrumento fue autoadministrado.

Para poder llevar a cabo este estudio, el mismo fue presentado y aprobado por el Comité de Investigación Interinstitucional del estado de Querétaro. Se consideraron los aspectos de confidencialidad y otorgamiento de consentimiento informado como parte de los criterios éticos.

La recolección final de datos se hizo con autorización de las autoridades de la institución educativa; de la misma manera, se solicitó el permiso a los docentes que se encontraban con cada grupo, para poder aplicar la encuesta. A los alumnos, se les explicó en qué consistía el estudio y se les solicitó su participación voluntaria.

Los resultados fueron capturados en una base de datos de SPSS v. 17, la muestra quedó constituida por un total de 188 alumnos encuestados. Se aplicó estadística descriptiva para llevar a cabo el procesamiento de los datos.

\section{Resultados}

De los 188 alumnos encuestados, se encontró que el promedio de edad fue de 22.4 años, con una desviación estándar de \pm 2.97 . El $85 \%$ de ellos ha vivido la muerte de un familiar cercano o amigo y $78.19 \%$ ha tenido la experiencia de ver morir a un paciente hospitalizado, encontrando que más del $75 \%$ de los alumnos de cada grupo incluido en el estudio, ha vivido este fenómeno. Con respecto a esto último, el $46 \%$ de los estudiantes refirió que no se consideran preparados para este proceso y el $75 \%$ hizo mención que no sabría cómo apoyar al familiar de un paciente que ha fallecido.

Con respecto a la concepción sobre la muerte, $59 \%$ de los estudiantes refiere que esta es el proceso natural que todos los seres humanos deben vivir y que es el cese definitivo e irreversible de las funciones de los sistemas vitales (51\%).

A continuación, se desglosan los resultados más relevantes del estudio, clasificándolos en aquellos referentes a experiencias y los que tienen que ver con conocimientos.

\section{Experiencias sobre la muerte}

Se plantearon preguntas sobre el actuar de los estudiantes al presentarse el fenómeno, de manera tal que $67 \%$ refirieron que cuando saben que un paciente va a morir, buscan que el paciente esté cómodo dentro de lo posible; $13.8 \%$ buscaban hablar más con él mismo, sin embargo, $5.8 \%$ se alejaron o mostraron indiferencia. En relación a los sentimientos que los jóvenes han experimentado al ver morir a un paciente, los resultados se muestran en la figura 1, la cual expone que 108 (57\%) jóvenes manifiestan sentimientos de inconformidad ante el fenómeno del morir de un paciente, aspecto en el que habría que ahondar, puesto que el $85 \%$ ya ha vivido esa experiencia de manera directa o indirecta con familiares y amigos, lo que puede generar sentimientos de fortaleza o debilidad; y poco más de la tercera parte, el $35 \%$ no contestaron o experimentaron otro sentimiento, lo que pudiera ser indicativo que ni siquiera saben verbalizarlo.

Sobre el "qué hacer si un paciente expresa que tiene miedo de morir", se obtuvieron respuestas diversas: $37 \%$ señaló

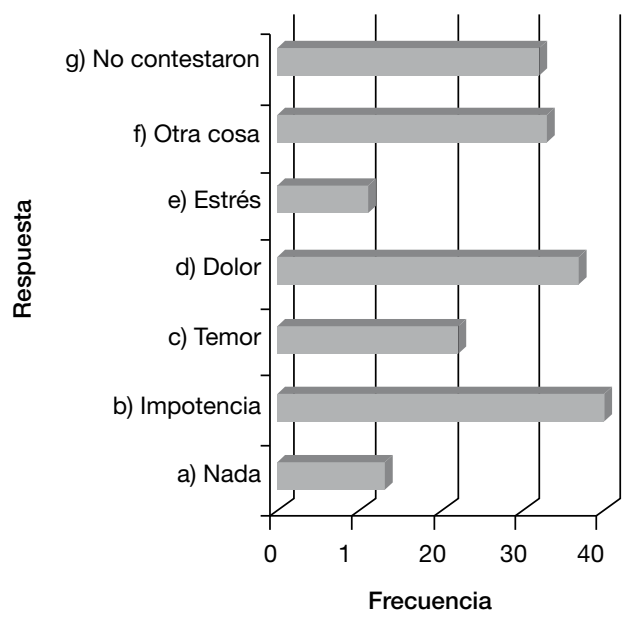

Figura 1 Sentimientos de los estudiantes ante la experiencia del ver morir a un paciente.

que tratarían de hacer que el paciente sobrellevara su situación, $21 \%$ indicaron que muy probablemente llorarían y $17 \%$ refirió que simplemente no sabría qué hacer. Muy vinculadas con lo anterior, se encontró que $44.1 \%$ sí se consideran capaces de superar un proceso de muerte de un familiar o un paciente, pero el $11 \%$ señaló lo contrario y el $31.4 \%$ indicó que les costaría mucho sobrellevarlo.

Dentro de las preguntas vinculadas con la experiencia, se cuestionó la percepción de los jóvenes sobre el personal de enfermería que labora en los lugares a donde llevaron a cabo sus prácticas y el proceso de la muerte, manifestando estos que el personal de enfermería no está preparado para "manejar" la muerte del paciente $(38 \%)$ o bien, es indiferente ante el fenómeno (21.8\%).

De la misma manera, el $35.1 \%$ considera que el personal ve el proceso de la muerte como algo complicado, ya que no se le ha enseñado cómo actuar. Al respecto, $48.4 \%$ de los estudiantes opinan que les gustaría que el personal fuera más solidario en el proceso del morir del paciente y $43.08 \%$ que el personal mostrara más apoyo al familiar del paciente moribundo. Por último, $77.65 \%$ de los estudiantes opina que el personal de enfermería de las instituciones hospitalarias ya no siente dolor ante la muerte de un paciente, dado que está acostumbrado a vivir continuamente esta experiencia.

En relación a si los estudiantes sienten temor a la muerte, las opiniones fueron divididas, ya que el $51.5 \%$ señalaron que si y el resto que no. Al mismo tiempo, para los participantes, hablar de la muerte resulta un tema interesante en el $37.2 \%$, y por otra parte, un tema natural en el $36.7 \%$.

\section{Conocimientos sobre el proceso de la muerte}

El $42.5 \%$ de los alumnos consideran que tienen un deficiente conocimiento sobre los aspectos de tanatología; relacionado con esto, el $79.78 \%$ señala que cuando un paciente va a fallecer, buscan darle un buen cuidado y/o calidad de vida.

Se asume que la tanatología es la ciencia y arte del bien morir (83\%), y que el proceso de duelo implica respetar el dolor de la pérdida del ser querido (26.1\%). Al respecto del duelo, el $75 \%$ señala que este proceso incluye las etapas 
de negación, ira, negociación, depresión y aceptación. El $42.6 \%$ indicó que el proceso de duelo incluye sentimientos de hostilidad, depresión, autorechazo y alteraciones en las relaciones familiares; el $23.4 \%$ indicó que sólo hay depresión.

El 61.2\% de los alumnos mencionó que el proceso de duelo de una persona depende de aspectos familiares, características de la muerte del paciente, tiempo en que se dio el fallecimiento y la situación psicológica de la persona; el $28.19 \%$ señaló que sólo es esto último.

Casi el total de los estudiantes (94.7\%) consideró que, es necesario llevar temáticas de tanatología durante la formación universitaria.

\section{Discusión}

La muerte, aún y cuando se le conceptualiza como un fenómeno natural, no deja de ser una experiencia impactante cuya realidad no siempre es aceptada en su totalidad, dado que no ha dejado de ser un hecho temido por la mayor parte de las personas.

Los resultados obtenidos a través de este estudio denotan que los estudiantes no se sienten capaces de dar apoyo al paciente moribundo y a su familia, puesto que refieren un deficiente conocimiento en tanatología, esto a pesar de que la mayor parte de ellos ya han experimentado la muerte de una persona cercana, lo que lo hace un fenómeno conocido, aunque claramente es vivido de manera distinta en el ámbito hospitalario.

Lo anterior evidencia la insuficiente información que los estudiantes reciben para el desarrollo de recursos en la atención en este tipo de situaciones o bien, como señala González, Salvadores y Venturini ${ }^{15}$, se puede estar viviendo el fenómeno de que, como enfermería está tan acostumbrado a convivir con la muerte, concibe que los pacientes no podrían morir.

Aunado a lo anterior se tiene que las demandas de atención en salud, en el contexto actual, exigen un trato más humanizado, en especial cuando se trata de la experiencia vivida del paciente y su familia frente a un desenlace inevitable como lo es la muerte. De ahí, que se considere necesario evaluar la pertinencia de insertar contenidos sobre esta temática dentro de la currícula de la Licenciatura en Enfermería para proporcionar al estudiante los conocimientos necesarios, que le permitan brindar un cuidado integral y al mismo tiempo le otorgue seguridad en la realización de las tareas encomendadas dentro de su práctica.

La afectación física y emocional que conlleva el fenómeno del dolor ante la pérdida de un ser humano no sólo afecta a quien lo vive sino a quien lo vivencia, como es el caso del profesional de enfermería. Los profesionales de enfermería cotidianamente se enfrentan a situaciones que conllevan el dolor, la cercanía a la muerte y la muerte en sí misma como fin último de la vida. El estrés y la angustia que circundan el diario devenir del cuidado enfermero junto al paciente moribundo y sus familiares, precisa de una formación específica para el colectivo de enfermería. Por tanto, es indispensable ocuparse de aquellos aspectos que dada su naturaleza, amenazan la estabilidad emocional de enfermería, por lo que la salud mental de los profesionales de enfermería debe ser cuidada desde su formación dentro de las instituciones de educación superior.

Lo anterior, tiene estrecho vínculo con lo manifestado por Pichardo y Diner', cuando explican que el personal de enfermería se protege de esta clase de fenómenos "guardando distancia", aspecto que también identificaron los alumnos de este estudio, en el personal que se encuentra en las instituciones hospitalarias.

Asimismo, se encontró que los estudiantes reconocen la necesidad de poseer una preparación en aspectos vinculados con el proceso del duelo y la muerte, igual y como lo indica Maza ${ }^{10}$, de manera tal que el profesional posea conocimientos y actitudes basadas en principios éticos.

Por ello, es indispensable establecer mecanismos de intervención con el propósito de brindar apoyo psicológico para minimizar el impacto emocional que este tipo de sucesos ocasiona, e implementar acciones tendientes a brindar capacitación específica que les permita a los estudiantes desarrollar recursos personales para afrontar el estrés y la ansiedad que ocasiona este tipo de situaciones, que en más de las veces resultan difíciles de aceptar.

\section{Conflicto de intereses}

Los autores declaran no tener ningún conflicto de intereses.

\section{Financiamiento}

No se recibió patrocinio para llevar a cabo este artículo.

\section{Referencias}

1. Da Silva Brêtas JR, De Oliveira JR, Yamaguti L. Reflexões de estudantes de enfermagem sobre morte eo morrer. Rev Esc Enferm USP 2006;40(4):477-483.

2. Lértora-Mendoza C. Visiones filosóficas de la muerte. Agnición 2010;4:1-7.

3. Safatle V. $\mathrm{O}$ amor é mais frio que a morte: negatividade, infinitude e indeterminação na teoria Hegeliana do desejo. Kriterion, Belo Horizonte 2008;49(117):95-125.

4. De Martins Custodio MR. O processo de morte e morrer no enfoque dos acadêmicos de enfermagem. Encontro: Revista de Psicología 2010;13(18):127-142.

5. Assumpção EA. Tanatologia - Ciência da vida e da morte. In: Anais Do 1o. Congresso de Tanatologia e Bioética. Belo Horizonte: Sotamig, 2003; P. 21-36. Citado en: Lima-Neto VB. Tanatologia e logoterapia: um diálogo ontológico. Revista logos \& existência 2012;1(1):38-49.

6. Benbunan Bentata B, Cruz-Quintana F, Roa-Venegas JM, et al. Afrontamiento del dolor y la muerte en estudiantes de enfermería: una propuesta de intervención. International journal of clinical and health psychology 2007;7(1):197-205.

7. Pichardo-García LM, Diner K. La experiencia de la muerte y los cuidados paliativos. Una visión desde la enfermería. An Med (Mex) 2010;55(3):161-166.

8. Bifulco VA, Lochida LC. A formação na graduação dos profissionais de saúde e a educação para o cuidado de pacientes fora de recursos terapêuticos de cura. Revista Brasileira de Educação Médica 2009;33(1):92-100.

9. Aguilar-Pérez MG, Del Carmen-Dorantes P, Ortega-Pérez JR. Muerte y subjetividad: reflexiones a propósito de la formación de los profesionales en salud. Revista CES Psicología 2010;3(1):83-98. 
10. Maza-Cabrera M, Zavala-Gutiérrez M, Merino-Escobar JM. Actitud del profesional de enfermería ante la muerte de pacientes. Ciencia y enfermería (On Line) 2009;15(1):39-48.

11. Fernández AR. Feeling nurse after the passing of a loved one. Investigación y Educación en Enfermería 2010;28(2):267-74

12. Martínez-Torres MY, Flores-Bravo MC, Córdoba-Ávila MÁ, et al. Evaluación del conocimiento sobre cuidados tanatológicos y su aplicación por el personal de enfermería de un Centro Médico Nacional. Rev CONAMED 2010;15(1):4-14.
13. Carvalho LS, Oliveira MA, Portela SC, et al. A morte e o morrer no cotidiano de estudantes de Enfermagem. Rev Enferm UERJ 2006;14:551-557.

14. Moliner M. Diccionario de uso del español. 2da edición. Madrid: Gredos; 2004.

15. Chocarro-González L, González-Fernández R, Salvadores-Fuentes $\mathrm{P}$, et al. Negación de la muerte y su repercusión en los cuidados. Medipal 2012;19(4):148-154. 